\title{
HYBRID CENTRAL-UPWIND SCHEMES FOR NUMERICAL RESOLUTION OF TWO-PHASE FLOWS
}

\author{
Steinar Evje ${ }^{1}$ And TORE FlåtTen ${ }^{1}$
}

\begin{abstract}
In this paper we present a methodology for constructing accurate and efficient hybrid central-upwind (HCU) type schemes for the numerical resolution of a two-fluid model commonly used by the nuclear and petroleum industry. Particularly, we propose a method which does not make use of any information about the eigenstructure of the Jacobian matrix of the model. The two-fluid model possesses a highly nonlinear pressure law. From the mass conservation equations we develop an evolution equation which describes how pressure evolves in time. By applying a quasi-staggered Lax-Friedrichs type discretization for this pressure equation together with a Modified Lax-Friedrich type discretization of the convective terms, we obtain a central type scheme which allows to cope with the nonlinearity (nonlinear pressure waves) of the two-fluid model in a robust manner. Then, in order to obtain an accurate resolution of mass fronts, we employ a modification of the convective mass fluxes by hybridizing the central type mass flux components with upwind type components. This hybridization is based on a splitting of the mass fluxes into components corresponding to the pressure and volume fraction variables, recovering an accurate resolution of a contact discontinuity. In the numerical simulations, the resulting HCU scheme gives results comparable to an approximate Riemann solver while being superior in efficiency. Furthermore, the HCU scheme yields better robustness than other popular Riemann-free upwind schemes.
\end{abstract}

Mathematics Subject Classification. 35L65, 65M12, 76N10, 76T10.

Received: April 23, 2004.

\section{INTRODUCTION}

In this paper we investigate a 1-dimensional averaged model for two-phase flow in pipelines, in widespread use by the nuclear $[2,17]$ and petroleum industry $[3,11]$. This model is characterized by having eigenvalues that may take extremely different values; the sonic velocities are typically several orders of magnitude higher than the wave velocities corresponding to mass transport.

For this reason, simple flux vector splitting (FVS) or central schemes typically give a poor numerical approximation of the slow-moving waves for this model, as the numerical dissipation of such schemes must be adapted to the fastest waves (see for instance [8]). To achieve a high degree of accuracy for all waves, the numerical dissipation mechanism must somehow be adapted to each wave phenomenon separately. Although approximate Riemann solvers like the Roe scheme of Toumi et al. [5,21,22] are robust and give accurate results for all waves,

\footnotetext{
Keywords and phrases. Two-phase flow, two-fluid model, hyperbolic system of conservation laws, central discretization, upwind discretization, pressure evolution equation, hybrid scheme.

1 RF-Rogaland Research, Prof. Olav Hanssensvei 15, Stavanger, Norway. steinar.evje@rf .no; t.h.flatten@cma.uio.no
} 
they are highly CPU-demanding due to the complexity of the eigenstructure of the model [8]. In particular, no tractable analytical expressions exist for the eigenvalues, which typically must be calculated numerically or from power series approximations. From an efficiency point of view, schemes that are independent of the eigenstructure are desirable.

A hybrid flux-splitting strategy denoted as AUSM (Advection Upstream Splitting Methods) has been popular in the field of multiphase flow due to its simplicity and accuracy $[6,13,14]$. Here the basic idea is to solve each conservation equation separately, by splitting the cell interface velocity into left and right components where the effects of sonic waves are taken into account.

For the current model, such strategies have been investigated by Paillère et al. [15] who considered the $\mathrm{AUSM}^{+}$strategy of Liou [12], and Evje and Flåtten [8] who considered the AUSMD/V strategy of Wada and Liou [24]. Not requiring a full eigenstructure decomposition of the system, these schemes were nevertheless demonstrated to give an accurate description of all wave phenomena for several benchmark problems. However, some drawbacks are observed:

- As the numerical couplings between the various equations are loose, spurious oscillations are typically observed in the (slow) volume fraction waves.

- The schemes indirectly rely on the eigenstructure of the model through the use of a numerical sound velocity, which for two-phase mixtures may vary depending of the form of additional closure terms (interphasic momentum exchange terms).

In this paper, we construct a Riemann-free numerical scheme which avoids these drawbacks. Our approach is as follows: the main difficulty with the model we consider is the nonlinearity associated with the pressure law. In order to obtain information about this part of the flux, particularly how it is related to other variables, we develop a dynamic equation describing how pressure evolves in time. This pressure evolution equation is obtained from the mass conservation equations. Then we proceed along the two following steps:

(1) First, we construct a central type scheme based on a splitting of the system into a convective and a pressure part. A robust numerical flux is obtained for the pressure part by employing a quasi-staggered Lax-Friedrichs type discretization of the pressure evolution equation at the cell interfaces. Similarly, a related central type discretization approach is used for the convective terms. More precisely, these fluxes correspond to those used in the Modified Lax-Friedrich scheme [19]. The resulting central type scheme ensures a robust treatment of the nonlinear pressure waves.

(2) Second, in order to ensure that accurate resolution of mass fronts is obtained we hybridize the convective central fluxes with corresponding simple upwind fluxes (which employ only the fluid velocity) so that we recover the upwind flux for a moving or stationary contact discontinuity. This hybridization is achieved by splitting the mass fluxes into a pressure and volume fraction part, and automatically enforces a strong numerical coupling between the gas and liquid mass conservation equation.

On several benchmark cases, this simple procedure gives accuracy and robustness properties comparable to the much more computationally demanding Roe scheme. In addition, improved stability properties over the AUSM strategy are clearly observed.

The paper is organized as follows: in Section 2 we present the two-fluid model we will be working with. In Section 3 we derive an evolution equation for the pressure. In Section 4 we present the central scheme that forms the foundation for this paper. A key role is played by the pressure evolution equation which provides a robust numerical flux for the pressure term. In Section 5 we present the hybrid central-upwind (HCU) scheme. A main point of this paper lies in Section 5.1, where we derive the hybrid mass fluxes (40) and (41).

In Section 6 we verify that the HCU scheme possesses certain desirable properties. In Section 7 we apply it to a set of test cases found in the literature. Comparisons are made with an approximate Riemann solver (Roe scheme), as well as the AUSMD scheme considered in [8] and the $\mathrm{AUSM}^{+}$scheme considered in [15]. 


\section{The TWO-FLUID MODEL}

Throughout this paper we will be concerned with the common two-fluid model formulated by stating separate conservation equations for mass and momentum for the two fluids, which we will denote as a gas (g) and a liquid (l) phase. We choose a formulation of the model that has been widely studied in the scientific literature $[4,5,8,15,22]$. We let $\mathbf{U}$ be the vector of conserved variables

$$
\mathbf{U}=\left[\begin{array}{c}
\rho_{\mathrm{g}} \alpha_{\mathrm{g}} \\
\rho_{\mathrm{l}} \alpha_{\mathrm{l}} \\
\rho_{\mathrm{g}} \alpha_{\mathrm{g}} v_{\mathrm{g}} \\
\rho_{\mathrm{l}} \alpha_{\mathrm{l}} v_{\mathrm{l}}
\end{array}\right]=\left[\begin{array}{c}
m_{\mathrm{g}} \\
m_{\mathrm{l}} \\
I_{\mathrm{g}} \\
I_{\mathrm{l}}
\end{array}\right]
$$

By using the notation $\Delta p=p-p^{\mathrm{i}}$, where $p^{\mathrm{i}}$ is the interfacial pressure, the model can be written on the form

- Conservation of mass

- Conservation of momentum

$$
\begin{gathered}
\frac{\partial}{\partial t}\left(\rho_{\mathrm{g}} \alpha_{\mathrm{g}}\right)+\frac{\partial}{\partial x}\left(\rho_{\mathrm{g}} \alpha_{\mathrm{g}} v_{\mathrm{g}}\right)=0, \\
\frac{\partial}{\partial t}\left(\rho_{\mathrm{l}} \alpha_{\mathrm{l}}\right)+\frac{\partial}{\partial x}\left(\rho_{\mathrm{l}} \alpha_{\mathrm{l}} v_{\mathrm{l}}\right)=0,
\end{gathered}
$$

$$
\begin{gathered}
\frac{\partial}{\partial t}\left(\rho_{\mathrm{g}} \alpha_{\mathrm{g}} v_{\mathrm{g}}\right)+\frac{\partial}{\partial x}\left(\rho_{\mathrm{g}} \alpha_{\mathrm{g}} v_{\mathrm{g}}^{2}\right)+\Delta p \frac{\partial}{\partial x}\left(\alpha_{\mathrm{g}}\right)+\alpha_{\mathrm{g}} \frac{\partial}{\partial x}(p)=Q_{\mathrm{g}}+M_{\mathrm{g}}^{\mathrm{D}}, \\
\frac{\partial}{\partial t}\left(\rho_{1} \alpha_{1} v_{\mathrm{l}}\right)+\frac{\partial}{\partial x}\left(\rho_{1} \alpha_{1} v_{1}^{2}\right)+\Delta p \frac{\partial}{\partial x}\left(\alpha_{1}\right)+\alpha_{1} \frac{\partial}{\partial x}(p)=Q_{1}+M_{1}^{\mathrm{D}} .
\end{gathered}
$$

Here $\alpha_{k}$ is the volume fraction of phase $k$ with

$$
\alpha_{\mathrm{g}}+\alpha_{\mathrm{l}}=1
$$

$\rho_{k}$ and $v_{k}$ denote the density and fluid velocity of phase $k$, and $p$ is the pressure common to both phases. $M_{k}^{\mathrm{D}}$ represents interfacial drag force with $M_{\mathrm{g}}^{\mathrm{D}}+M_{1}^{\mathrm{D}}=0$ whereas $Q_{k}$ represent source terms due to gravity, friction, etc. For reasons of clarity and consistency with previous works, we assume a nondifferential interfacial drag force $M_{k}^{\mathrm{D}}=M_{k}^{\mathrm{D}}(\mathbf{U})$.

\subsection{Submodels}

The system is closed by some equation of states (EOS) for the liquid and gas phase. The numerical methods we study in this work allow general expressions for the EOS. However, for the numerical simulations presented in this work we follow [8] and assume the simplified thermodynamic relations

$$
\rho_{1}=\rho_{1,0}+\frac{p-p_{0}}{a_{1}^{2}}
$$

and

where

$$
\rho_{\mathrm{g}}=\frac{p}{a_{\mathrm{g}}^{2}}
$$

$$
\begin{aligned}
p_{0} & =1 \mathrm{bar}=10^{5} \mathrm{~Pa} \\
\rho_{\mathrm{l}, 0} & =1000 \mathrm{~kg} / \mathrm{m}^{3}, \\
a_{\mathrm{g}}^{2} & =10^{5}(\mathrm{~m} / \mathrm{s})^{2} \\
a_{\mathrm{l}} & =10^{3} \mathrm{~m} / \mathrm{s} .
\end{aligned}
$$


The models (7) and (8) correspond to a general stiffened gas EOS of the form

$$
p=\left(\gamma_{k}-1\right) a_{k}^{2} \rho_{k}-\gamma_{k} \pi_{k},
$$

where $\pi_{k}=\left(a_{k}^{2} \rho_{k, 0}-p_{0}\right) / 2$ where $\rho_{k, 0}$ represents the material density and $p_{0}$ the ambient pressure. $\gamma_{k}$ and $\pi_{k}$ are constants specific for each phase. Particularly, by choosing $\gamma_{1}=2$ we recover (7) while (8) is obtained by choosing $\gamma_{\mathrm{g}}=2$ and $\pi_{\mathrm{g}}=0$.

Moreover, we will treat $Q_{k}$ as a pure source term, assuming that it does not contain any differential operators. We use the interface pressure correction

$$
\Delta p=\sigma \frac{\alpha_{\mathrm{g}} \alpha_{\mathrm{l}} \rho_{\mathrm{g}} \rho_{\mathrm{l}}}{\rho_{\mathrm{g}} \alpha_{\mathrm{l}}+\rho_{\mathrm{l}} \alpha_{\mathrm{g}}}\left(v_{\mathrm{g}}-v_{\mathrm{l}}\right)^{2} .
$$

If the relative velocity between the phases is low, the choice $\sigma>1$ ensures that the model is a hyperbolic system of conservation laws. For the purposes of this paper, we set $\sigma=1.2$.

For a more detailed analysis of the hyperbolicity condition, see for instance $[5,8]$.

\subsection{Conservative and primitive variables}

Having solved for the conservative variable $\mathbf{U}$, we need to obtain the primitive variables $\left(\alpha_{\mathrm{g}}, p, v_{\mathrm{g}}, v_{\mathrm{l}}\right)$. For the pressure variable we see that by writing the volume fraction equation (6) in terms of the conserved variables as

$$
\frac{m_{\mathrm{g}}}{\rho_{\mathrm{g}}(p)}+\frac{m_{\mathrm{l}}}{\rho_{\mathrm{l}}(p)}=1,
$$

we obtain a relation yielding the pressure $p\left(m_{\mathrm{g}}, m_{\mathrm{l}}\right)$. Using the relatively simple form of EOS given by $(7)$ and (8) we see that the pressure $p$ is found as a positive root of a second order polynomial. For more general EOS we must solve a non-linear system of equations, for instance by using a Newton-Rapson algorithm. Moreover, the fluid velocities $v_{\mathrm{g}}$ and $v_{\mathrm{l}}$ are obtained directly from the relations

$$
v_{\mathrm{g}}=\frac{U_{3}}{U_{1}}, \quad v_{1}=\frac{U_{4}}{U_{2}} .
$$

Remark 1. It should be noted that the model (2)-(5) assumes that dynamic energy transfers can be neglected; consequently a static temperature or entropy distribution is implicitly assumed and the EOS can always be expressed as a one-variable function only, i.e. $\rho_{k}=\rho_{k}(p)$ for phase $k$.

We emphasize that the methods we develop do not require simple linear relations as given by (7) and (8). Nonlinear EOS have been tested in [10]. The only complication added by using a general nonlinear EOS is the resolution algorithm which determines the pressure from the general relation (10). Note that even with linear relations like (7) and (8) the pressure law $p=p\left(m_{\mathrm{g}}, m_{\mathrm{l}}\right)$ becomes nonlinear as a function of $m_{\mathrm{g}}$ and $m_{\mathrm{l}}$.

\section{NumERICAL SCHEMES}

In the following, we will find it convenient to split the fluxes into convective and pressure parts and deal with each term separately. We write the system (2)-(5) as follows

$$
\frac{\partial \mathbf{U}}{\partial t}+\frac{\partial \mathbf{F}_{\mathrm{c}}}{\partial x}+\Delta p \frac{\partial \mathbf{H}}{\partial x}+\mathbf{H} \frac{\partial p}{\partial x}=\mathbf{Q},
$$

where

$$
\mathbf{Q}=\left[\begin{array}{c}
0 \\
0 \\
Q_{\mathrm{g}} \\
Q_{1}
\end{array}\right], \quad \mathbf{H}=\left[\begin{array}{c}
0 \\
0 \\
\alpha_{\mathrm{g}} \\
\alpha_{1}
\end{array}\right] \text { and } \mathbf{F}_{\mathrm{c}}=\left[\begin{array}{c}
\rho_{\mathrm{g}} \alpha_{\mathrm{g}} v_{\mathrm{g}} \\
\rho_{\mathrm{l}} \alpha_{\mathrm{l}} v_{\mathrm{l}} \\
\rho_{\mathrm{g}} \alpha_{\mathrm{g}} v_{\mathrm{g}}^{2} \\
\rho_{\mathrm{l}} \alpha_{\mathrm{l}} v_{1}^{2}
\end{array}\right]
$$


In this paper we will consider discrete schemes on the form

$$
\frac{\mathbf{U}_{j}^{n+1}-\mathbf{U}_{j}^{n}}{\Delta t}+\frac{\left[\mathbf{F}_{\mathrm{c}}\right]_{j+1 / 2}^{n}-\left[\mathbf{F}_{\mathrm{c}}\right]_{j-1 / 2}^{n}}{\Delta x}+\Delta p_{j}^{n} \frac{\mathbf{H}_{j+1 / 2}^{n}-\mathbf{H}_{j-1 / 2}^{n}}{\Delta x}+\mathbf{H}_{j}^{n} \frac{P_{j+1 / 2}^{n+1}-P_{j-1 / 2}^{n+1}}{\Delta x}=\mathbf{Q}_{j}^{n} .
$$

In the next section we will specify the numerical fluxes $\left[\mathbf{F}_{\mathrm{c}}\right]_{j+1 / 2}^{n}, \mathbf{H}_{j+1 / 2}^{n}$, and $P_{j+1 / 2}^{n+1}$.

\subsection{Some useful differential relations and a pressure evolution equation}

For later use we here derive some useful differential relations. By using the relation (10), we can derive the differentials

$$
\mathrm{d} p=\kappa\left(\rho_{\mathrm{l}} \mathrm{d} m_{\mathrm{g}}+\rho_{\mathrm{g}} \mathrm{d} m_{\mathrm{l}}\right)
$$

and

$$
\mathrm{d} \alpha_{1}=\kappa\left(\alpha_{\mathrm{g}} \frac{\partial \rho_{\mathrm{g}}}{\partial p} \mathrm{~d} m_{\mathrm{l}}-\alpha_{\mathrm{l}} \frac{\partial \rho_{\mathrm{l}}}{\partial p} \mathrm{~d} m_{\mathrm{g}}\right)
$$

where

$$
\kappa=\frac{1}{\frac{\partial \rho_{\mathrm{l}}}{\partial p} \alpha_{\mathrm{l}} \rho_{\mathrm{g}}+\frac{\partial \rho_{\mathrm{g}}}{\partial p} \alpha_{\mathrm{g}} \rho_{\mathrm{l}}} .
$$

Furthermore, we can write the mass differentials in terms of the pressure and volume fraction differentials as follows:

$$
\mathrm{d} m_{\mathrm{g}}=\alpha_{\mathrm{g}} \frac{\partial \rho_{\mathrm{g}}}{\partial p} \mathrm{~d} p-\rho_{\mathrm{g}} \mathrm{d} \alpha_{1}
$$

and

$$
\mathrm{d} m_{\mathrm{l}}=\alpha_{1} \frac{\partial \rho_{\mathrm{l}}}{\partial p} \mathrm{~d} p+\rho_{\mathrm{l}} \mathrm{d} \alpha_{\mathrm{l}}
$$

By using the differential (13) and the mass equations (2) and (3) we obtain the dynamical relation

$$
\frac{\partial p}{\partial t}+\kappa\left(\rho_{\mathrm{l}} \frac{\partial}{\partial x}\left(\rho_{\mathrm{g}} \alpha_{\mathrm{g}} v_{\mathrm{g}}\right)+\rho_{\mathrm{g}} \frac{\partial}{\partial x}\left(\rho_{\mathrm{l}} \alpha_{\mathrm{l}} v_{\mathrm{l}}\right)\right)=0
$$

This equation gives us an evolution equation for the pressure which provides useful information how the pressure is related to other variables. A main idea in this paper is to construct a numerical flux for the pressure term based on this equation.

\section{A Central SCHEME}

With the above relations in hand we will now describe a central scheme consisting of basically two main steps; the first step deals with the calculation of the cell interface pressure $P_{j+1 / 2}$ from (18) whereas the second deals with the calculation of the cell center pressure $p_{j}$ from the masses $m_{k, j}$ via the pressure law $p=p\left(m_{\mathrm{g}}, m_{\mathrm{l}}\right)$ defined by (10). The essential part of the second step is to develop numerical convective fluxes which are consistent with the pressure splitting $P_{j+1 / 2}$. In this manner, by making a temporal coupling between the pressure and velocity fields, we achieve the following two benefits:

- A coherent time development is attained for the cell interface pressure $P_{j+1 / 2}$ and the cell center pressure $p_{j}\left(m_{\mathrm{g}}, m_{1}\right)$, ensuring a high degree of robustness.

- The pressure--momentum coupling naturally allows for implicit versions of the scheme.

In this paper we explore properties of an explicit scheme. In [9] the pressure-momentum coupling was exploited to construct implicit versions of the AUSMD scheme investigated in [8]. 


\subsection{Step I: specification of the pressure flux $\boldsymbol{p}_{\boldsymbol{j}+\mathbf{1} / \mathbf{2}}$}

Discretizing the equation (18) by a staggered Lax-Friedrichs type of scheme at the cell interface $x_{j+1 / 2}$, we obtain

$$
\frac{p_{j+1 / 2}^{n+1}-\frac{1}{2}\left(p_{j}^{n}+p_{j+1}^{n}\right)}{\Delta t}=-\left(\kappa \rho_{\mathrm{l}}\right)_{j+1 / 2}^{n} \frac{I_{\mathrm{g}, j+1}^{n}-I_{\mathrm{g}, j}^{n}}{\Delta x}-\left(\kappa \rho_{\mathrm{g}}\right)_{j+1 / 2}^{n} \frac{I_{1, j+1}^{n}-I_{1, j}^{n}}{\Delta x}
$$

where we use the shorthand

$$
I_{k}=m_{k} v_{k}
$$

Note that the cell interface pressure $p_{j+1 / 2}^{n+1}$ can be written on the viscous form

$$
\begin{aligned}
p_{j+1 / 2}^{n+1} & =P_{j+1 / 2}=P\left(\mathbf{U}_{j}^{n}, \mathbf{U}_{j+1}^{n}\right) \\
& =\frac{1}{2}\left(p_{j}^{n}+p_{j+1}^{n}\right)-\left[D_{\mathrm{g}, j+1 / 2}^{n}\left(I_{\mathrm{g}, j+1}^{n}-I_{\mathrm{g}, j}^{n}\right)+D_{1, j+1 / 2}^{n}\left(I_{1, j+1}^{n}-I_{1, j}^{n}\right)\right]
\end{aligned}
$$

where the numerical viscosity coefficients $D_{k, j+1 / 2}^{n}$ are given by

$$
D_{\mathrm{g}, j+1 / 2}^{n}=\frac{\Delta t}{\Delta x} \kappa_{j+1 / 2}^{n} \rho_{\mathrm{l}, j+1 / 2}^{n}
$$

and

$$
D_{1, j+1 / 2}^{n}=\frac{\Delta t}{\Delta x} \kappa_{j+1 / 2}^{n} \rho_{\mathrm{g}, j+1 / 2}^{n},
$$

where $\rho_{\mathrm{l}}, \rho_{\mathrm{g}}$, and $\kappa$ are given by $(7),(8)$, and (15). To calculate interface values $(\cdot)_{j+1 / 2}^{n}$ needed for the coefficients (21) and (22) we propose using the following averages

and

$$
\begin{aligned}
\alpha_{j+1 / 2}^{n} & =\frac{1}{2}\left(\alpha_{j}^{n}+\alpha_{j+1}^{n}\right), \\
\rho_{j+1 / 2}^{n} & =\frac{1}{2}\left(\rho_{j}^{n}+\rho_{j+1}^{n}\right)
\end{aligned}
$$

$$
\left(\frac{\partial \rho}{\partial p}\right)_{j+1 / 2}= \begin{cases}\frac{\rho_{j+1}-\rho_{j}}{p_{j+1}-p_{j}} & \text { if } p_{j} \neq p_{j+1} \\ \left(\frac{\partial \rho}{\partial p}\right)_{j} & \text { otherwise. }\end{cases}
$$

Thus, these coefficients do not require a reevaluation of the EOS at the cell interface.

Remark 2. We note that the role of the pressure evolution equation (19) is simply to define an appropriate numerical pressure $P_{j+1 / 2}=P\left(\mathbf{U}_{j}, \mathbf{U}_{j+1}\right)$ for the discretization of the pressure term in (12). From (20) we easily see that this numerical pressure is consistent with the physical pressure, i.e. $P(\mathbf{U}, \mathbf{U})=p$ for all $\mathbf{U}$.

\subsection{Step II: specification of the convective fluxes $\left(\rho_{k} \alpha_{k} v_{k}\right)_{j+1 / 2}$ and $\left(\rho_{k} \alpha_{k} v_{k}^{2}\right)_{j+1 / 2}$}

\subsubsection{Convective mass fluxes}

We here aim to obtain a flux yielding a calculation of masses consistent with the pressure calculation described in the previous section, and by that ensure stable (non-oscillatory) approximations of the pressure variable. Going back to the pressure equation (19), we see that it naturally defines a conservative scheme for calculating masses at cell interface $j+1 / 2$ as

$$
\frac{m_{k, j+1 / 2}^{n+1}-\frac{1}{2}\left(m_{k, j}^{n}+m_{k, j+1}^{n}\right)}{\Delta t}+\frac{I_{k, j+1}^{n}-I_{k, j}^{n}}{\Delta x}=0 .
$$


If we now compute the simple average

$$
m_{k, j}^{n}=\frac{1}{2}\left(m_{k, j-1 / 2}^{n}+m_{k, j+1 / 2}^{n}\right)
$$

and substitute (26), we obtain the following difference equation for $m_{k, j}$

$$
\frac{m_{k, j}^{n+1}-\frac{1}{4}\left(2 m_{k, j}^{n}+m_{k, j-1}^{n}+m_{k, j+1}^{n}\right)}{\Delta t}+\frac{1}{2 \Delta x}\left(I_{k, j+1}^{n}-I_{k, j-1}^{n}\right)=0
$$

which can be written on flux-conservative form with the numerical fluxes

$$
\left(\rho_{k} \alpha_{k} v_{k}\right)_{j+1 / 2}^{n}=F_{k}^{\mathrm{C}}\left(\mathbf{U}_{j}^{n}, \mathbf{U}_{j+1}^{n}\right)=\frac{1}{2}\left(I_{k, j}^{n}+I_{k, j+1}^{n}\right)+\frac{1}{4} \frac{\Delta x}{\Delta t}\left(m_{k, j}^{n}-m_{k, j+1}^{n}\right) .
$$

Here the superscript "C" indicates that $F_{k}^{\mathrm{C}}$ is a central flux.

\subsubsection{Convective momentum fluxes}

To ensure consistency between the numerical dissipation for convective mass and momentum transport, we propose discretizing the convective momentum fluxes as

$$
\left(\rho_{k} \alpha_{k} v_{k}^{2}\right)_{j+1 / 2}^{n}=\frac{1}{2}\left(\left[\rho_{k} \alpha_{k} v_{k}^{2}\right]_{j}^{n}+\left[\rho_{k} \alpha_{k} v_{k}^{2}\right]_{j+1}^{n}\right)+\frac{1}{4} \frac{\Delta x}{\Delta t}\left(I_{k, j}^{n}-I_{k, j+1}^{n}\right),
$$

by the natural extension of (29).

Remark 3. Note that these central fluxes correspond exactly to the modified Lax-Friedrichs scheme studied by Tadmor [19], who formally demonstrated convergence results to the physically relevant entropy solution. In particular, he demonstrated that the modified Lax-Friedrichs scheme is TVD for scalar conservation laws and satisfies a discrete entropy inequality for all entropy-flux pairs associated with an arbitrary system of conservation laws.

\subsection{Remaining terms}

What remains to be calculated are the cell interface volume fractions $\mathbf{H}_{j+1 / 2}^{n}$ for discretization of the term $\Delta p \partial_{x} \alpha$. Consistent with the expression (23), we propose the simple central discretization

$$
\alpha_{j+1 / 2}^{n}=\frac{1}{2}\left(\alpha_{j}^{n}+\alpha_{j+1}^{n}\right)
$$

In this respect we follow in the footsteps of Coquel et al. [4] and Paillère et al. [15].

\section{Removal of NUMERICAL Dissipation}

As the numerical experiments in this paper show, the proposed central scheme is highly robust, and yields an adequate resolution of sonic waves. However, it is highly diffusive on the slow-moving volume fraction waves. In the following, we propose a mechanism for removing numerical dissipation based on studying the simple contact discontinuity given by

$$
\begin{gathered}
p_{\mathrm{L}}=p_{\mathrm{R}}=p \\
\alpha_{\mathrm{L}} \neq \alpha_{\mathrm{R}} \\
\left(v_{\mathrm{g}}\right)_{\mathrm{L}}=\left(v_{\mathrm{l}}\right)_{\mathrm{L}}=\left(v_{\mathrm{g}}\right)_{\mathrm{R}}=\left(v_{\mathrm{l}}\right)_{\mathrm{R}}=v .
\end{gathered}
$$


Now all pressure terms vanish from the model (2)-(5) and it is seen that the solution to this initial value problem is simply that the discontinuity will propagate with the velocity $v$. This exact solution then gives the upwind mass flux

and momentum flux

$$
(\rho \alpha v)_{j+1 / 2}=\frac{1}{2} \rho\left(\alpha_{\mathrm{L}}+\alpha_{\mathrm{R}}\right) v-\frac{1}{2} \rho\left(\alpha_{\mathrm{R}}-\alpha_{\mathrm{L}}\right)|v|
$$

$$
\left(\rho \alpha v^{2}\right)_{j+1 / 2}=\frac{1}{2} \rho v\left(\alpha_{\mathrm{L}}+\alpha_{\mathrm{R}}\right) v-\frac{1}{2} \rho v\left(\alpha_{\mathrm{R}}-\alpha_{\mathrm{L}}\right)|v|
$$

where we have used that $p_{\mathrm{L}}=p_{\mathrm{R}}$ implies $\rho_{\mathrm{L}}=\rho_{\mathrm{R}}$ (see Rem. 1).

We now wish to hybridize the central mass flux $F^{\mathrm{C}}(29)$ with an upwind flux $F^{\mathrm{U}}$ such that we recover the upwind flux (33) for the contact discontinuity (32). More specifically, we wish to combine $F^{\mathrm{C}}$ and $F^{\mathrm{U}}$ such that the central part is used for the flux component associated with pressure (which is constant across the contact) and the upwind part is used for the flux component associated with volume fraction (which is discontinuous across the contact). In the following, we will describe how to achieve this.

\subsection{Construction of hybrid mass fluxes}

We consider a general discretization of the mass equations

$$
\frac{m_{k, j}^{n+1}-m_{k, j}^{n}}{\Delta t}=-\frac{1}{\Delta x}\left(F_{k, j+1 / 2}^{n}-F_{k, j-1 / 2}^{n}\right)
$$

where $F_{k, j+1 / 2}=F_{k}\left(U_{j}, U_{j+1}\right)$ is the numerical mass flux at cell interface $x_{j+1 / 2}$ corresponding to the physical flux $f_{k}(U)=\rho_{k} \alpha_{k} v_{k}$. From (16) and (17) we see that the mass differentials $\mathrm{d} m_{k}$ can be splitted in a pressure component $\mathrm{d} p$ and a volume fraction component $\mathrm{d} \alpha$. We now want to design a numerical flux which is consistent with this splitting, i.e. we introduce auxiliary flux components $F_{p}$ and $F_{\alpha}$ such that the mass fluxes $F_{1}$ and $F_{\mathrm{g}}$ are given by

and

$$
F_{1}=\alpha_{1} \frac{\partial \rho_{1}}{\partial p} F_{p}+\rho_{1} F_{\alpha}
$$

$$
F_{\mathrm{g}}=\alpha_{\mathrm{g}} \frac{\partial \rho_{\mathrm{g}}}{\partial p} F_{p}-\rho_{\mathrm{g}} F_{\alpha}
$$

The flux component $F_{p}$ is associated with the pressure, hence we want to assign the central mass flux $F^{\mathrm{C}}$ for stable approximation of pressure for all waves. By the differential relation $(13), F_{p}$ naturally acquires the following form

$$
F_{p}=\kappa \rho_{\mathrm{g}} F_{\mathrm{l}}^{\mathrm{C}}+\kappa \rho_{\mathrm{l}} F_{\mathrm{g}}^{\mathrm{C}}
$$

Similarly, the flux component $F_{\alpha}$ is associated with the volume fraction, hence we want to assign an upwind mass flux $F^{\mathrm{U}}$. By the differential relation (14), $F_{\alpha}$ is naturally expressed as

$$
F_{\alpha}=\kappa \frac{\partial \rho_{\mathrm{g}}}{\partial p} \alpha_{\mathrm{g}} F_{\mathrm{l}}^{\mathrm{U}}-\kappa \frac{\partial \rho_{\mathrm{l}}}{\partial p} \alpha_{\mathrm{l}} F_{\mathrm{g}}^{\mathrm{U}}
$$

Here we note that a subscript $j+1 / 2$ is assumed on the fluxes and coefficients. Substituting (38) and (39) into (37) and (36) we obtain the final hybrid mass fluxes

$$
F_{\mathrm{l}}=\kappa\left(\rho_{\mathrm{g}} \alpha_{\mathrm{l}} \frac{\partial \rho_{\mathrm{l}}}{\partial p} F_{\mathrm{l}}^{\mathrm{C}}+\rho_{\mathrm{l}} \alpha_{\mathrm{g}} \frac{\partial \rho_{\mathrm{g}}}{\partial p} F_{\mathrm{l}}^{\mathrm{U}}+\rho_{\mathrm{l}} \alpha_{\mathrm{l}} \frac{\partial \rho_{\mathrm{l}}}{\partial p}\left(F_{\mathrm{g}}^{\mathrm{C}}-F_{\mathrm{g}}^{\mathrm{U}}\right)\right)
$$

and

$$
F_{\mathrm{g}}=\kappa\left(\rho_{\mathrm{l}} \alpha_{\mathrm{g}} \frac{\partial \rho_{\mathrm{g}}}{\partial p} F_{\mathrm{g}}^{\mathrm{C}}+\rho_{\mathrm{g}} \alpha_{\mathrm{l}} \frac{\partial \rho_{\mathrm{l}}}{\partial p} F_{\mathrm{g}}^{\mathrm{U}}+\rho_{\mathrm{g}} \alpha_{\mathrm{g}} \frac{\partial \rho_{\mathrm{g}}}{\partial p}\left(F_{\mathrm{l}}^{\mathrm{C}}-F_{\mathrm{l}}^{\mathrm{U}}\right)\right)
$$


The coefficient variables at $x_{j+1 / 2}$ remain to be determined. Consistent with the treatment of the coefficients of the pressure evolution equation (20) we suggest finding these from the relations (23)-(25).

Remark 4. We remark that the consistency criterion

$$
F_{k}(U, U)=f_{k}(U)=\rho_{k} \alpha_{k} v_{k}
$$

relating the numerical flux $F_{k}$ to the physical flux $f_{k}$, is satisfied for the hybrid fluxes (40) and (41) provided the fluxes $F_{k}^{\mathrm{U}}$ and $F_{k}^{\mathrm{C}}$ are consistent. In particular if $F_{k}^{\mathrm{U}}=F_{k}^{\mathrm{C}}$ the expressions (40) and (41) reduce to the trivial identity

$$
F_{k}=F_{k}^{\mathrm{U}}=F_{k}^{\mathrm{C}}
$$

\subsection{The $\boldsymbol{F}_{k}^{\mathrm{U}}$-component}

For the upwind mass flux $F_{k}^{\mathrm{U}}$, we propose to use an upwind flux investigated in [7] for a simpler two-phase flow model. That is, we define the cell interface velocity $v_{k, j+1 / 2}$ by

$$
v_{k, j+1 / 2}=\frac{1}{2}\left(v_{k, j}+v_{k, j+1}\right) .
$$

Now the mass flux is given by

$$
F_{k}^{\mathrm{U}}= \begin{cases}v_{k, j+1 / 2}\left(\rho_{k} \alpha_{k}\right)_{j} & \text { if } v_{k, j+1 / 2} \geq 0 \\ v_{k, j+1 / 2}\left(\rho_{k} \alpha_{k}\right)_{j+1} & \text { otherwise }\end{cases}
$$

It can easily be shown that this mass flux satisfies (33) for the contact discontinuity (32).

\subsection{Convective momentum fluxes}

For the convective momentum fluxes, we propose using the corresponding upwind momentum fluxes directly:

$$
\left(\rho_{k} \alpha_{k} v_{k}^{2}\right)_{j+1 / 2}= \begin{cases}v_{k, j+1 / 2}\left(\rho_{k} \alpha_{k} v_{k}\right)_{j} & \text { if } v_{k, j+1 / 2} \geq 0 \\ v_{k, j+1 / 2}\left(\rho_{k} \alpha_{k} v_{k}\right)_{j+1} & \text { otherwise }\end{cases}
$$

We may easily check that this momentum flux satisfies (34) for the contact discontinuity (32).

Remark 5. In general, one could consider using hybrid central-upwind fluxes also for the momentum terms, similar to the expressions (40) and (41), to take advantage of the robustness properties of the central scheme. However, as the numerical experiments of this paper show, using the simple upwind discretization (46) for the convective momentum fluxes directly gives adequate results.

\subsection{The HCU scheme}

We now summarize the numerical scheme just derived, referred to as the $H C U$ (Hybrid Central-Upwind) scheme. In the following, let $\delta_{x}$ be defined as

$$
\delta_{x} w_{j}=\frac{w_{j+1 / 2}-w_{j-1 / 2}}{\Delta x} .
$$




\section{Mass equations}

We discretize the mass equations as follows

$$
\frac{m_{k, j}^{n+1}-m_{k, j}^{n}}{\Delta t}=-\delta_{x} F_{k, j}^{n}
$$

where the mass fluxes $F_{k, j+1 / 2}$ are given by

$$
F_{\mathrm{l}}=\kappa\left(\rho_{\mathrm{g}} \alpha_{\mathrm{l}} \frac{\partial \rho_{\mathrm{l}}}{\partial p} F_{\mathrm{l}}^{\mathrm{C}}+\rho_{\mathrm{l}} \alpha_{\mathrm{g}} \frac{\partial \rho_{\mathrm{g}}}{\partial p} F_{\mathrm{l}}^{\mathrm{U}}+\rho_{\mathrm{l}} \alpha_{\mathrm{l}} \frac{\partial \rho_{\mathrm{l}}}{\partial p}\left(F_{\mathrm{g}}^{\mathrm{C}}-F_{\mathrm{g}}^{\mathrm{U}}\right)\right)
$$

and

$$
F_{\mathrm{g}}=\kappa\left(\rho_{\mathrm{l}} \alpha_{\mathrm{g}} \frac{\partial \rho_{\mathrm{g}}}{\partial p} F_{\mathrm{g}}^{\mathrm{C}}+\rho_{\mathrm{g}} \alpha_{\mathrm{l}} \frac{\partial \rho_{\mathrm{l}}}{\partial p} F_{\mathrm{g}}^{\mathrm{U}}+\rho_{\mathrm{g}} \alpha_{\mathrm{g}} \frac{\partial \rho_{\mathrm{g}}}{\partial p}\left(F_{\mathrm{l}}^{\mathrm{C}}-F_{\mathrm{l}}^{\mathrm{U}}\right)\right)
$$

as described in Section 5.1. Coefficients are determined from (23)-(25). Here

$$
F_{k, j+1 / 2}^{\mathrm{C}, n}=\frac{1}{2}\left(I_{k, j}^{n}+I_{k, j+1}^{n}\right)+\frac{1}{4} \frac{\Delta x}{\Delta t}\left(m_{k, j}^{n}-m_{k, j+1}^{n}\right), \quad m_{k}=\rho_{k} \alpha_{k}, \quad I_{k}=m_{k} v_{k}
$$

and

$$
F_{k, j+1 / 2}^{\mathrm{U}, n}=\left(\rho_{k} \alpha_{k} v_{k}\right)_{j+1 / 2}^{n}
$$

as described by (44) and (45).

\section{Momentum equations}

We discretize the momentum equations as follows

$$
\frac{I_{k, j}^{n+1}-I_{k, j}^{n}}{\Delta t}=-\delta_{x}\left(\rho_{k} \alpha_{k} v_{k}^{2}\right)_{j}^{n}-(\Delta p)_{j}^{n} \delta_{x}\left(\alpha_{k}\right)_{j}^{n}-\left(\alpha_{k}\right)_{j}^{n} \delta_{x}(P)_{j}^{n+1}+\left(Q_{\mathrm{g}}\right)_{j}^{n}
$$

where $(\Delta p)_{j}^{n}=\Delta p\left(\mathbf{U}_{j}^{n}\right)$ is determined from (9). Here

$$
\left(\alpha_{k}\right)_{j+1 / 2}^{n}=\frac{1}{2}\left(\alpha_{k, j}^{n}+\alpha_{k, j+1}^{n}\right)
$$

and

$$
P_{j+1 / 2}^{n+1}=\frac{1}{2}\left(p_{j}^{n}+p_{j+1}^{n}\right)-\Delta t\left(\kappa \rho_{\mathrm{l}}\right)_{j+1 / 2}^{n} \frac{I_{\mathrm{g}, j+1}^{n}-I_{\mathrm{g}, j}^{n}}{\Delta x}-\Delta t\left(\kappa \rho_{\mathrm{g}}\right)_{j+1 / 2}^{n} \frac{I_{1, j+1}^{n}-I_{1, j}^{n}}{\Delta x}
$$

as described in Section 4.1. Finally

$$
\left(\rho_{k} \alpha_{k} v_{k}^{2}\right)_{j+1 / 2}^{n}
$$

is given by (44) and (46). 


\section{Properties of the HCU SCheme}

In this section we verify that the proposed HCU scheme possesses desirable accuracy and stability properties.

\subsection{Accurate approximation of steady and moving contact discontinuities}

We revisit the contact discontinuity (32):

$$
\begin{gathered}
p_{\mathrm{L}}=p_{\mathrm{R}}=p \\
\alpha_{\mathrm{L}} \neq \alpha_{\mathrm{R}} \\
\left(v_{\mathrm{g}}\right)_{\mathrm{L}}=\left(v_{1}\right)_{\mathrm{L}}=\left(v_{\mathrm{g}}\right)_{\mathrm{R}}=\left(v_{1}\right)_{\mathrm{R}}=v .
\end{gathered}
$$

First, we note that the pressure will remain constant and uniform as the discontinuity is propagating. Consequently a natural requirement on a "good" flux $F_{k}^{\mathrm{C}}$ for stable pressure resolution is that it preserves the constancy of pressure for the moving or stationary contact discontinuity given by (32).

We write (13) as

where

$$
\mathrm{d} p=\kappa \mathrm{d} \mu
$$

$$
\mathrm{d} \mu=\rho_{\mathrm{g}} \mathrm{d} m_{\mathrm{l}}+\rho_{\mathrm{l}} \mathrm{d} m_{\mathrm{g}} .
$$

To maintain a constant pressure we must have $\mathrm{d} \mu=0$. Assuming constant pressure, (49) can be integrated to yield

$$
\mu=\rho_{\mathrm{g}} m_{\mathrm{l}}+\rho_{\mathrm{l}} m_{\mathrm{g}}=\rho_{\mathrm{g}} \rho_{\mathrm{l}}\left(\alpha_{\mathrm{l}}+\alpha_{\mathrm{g}}\right)=\rho_{\mathrm{g}} \rho_{\mathrm{l}} .
$$

To maintain constancy of $\mu$ and hence $p$ we now insist that the flux $F^{\mathrm{C}}$ is a consistent numerical flux when applied to the mix mass $\mu$. That is, we impose

$$
\rho_{\mathrm{g}} F_{\mathrm{l}}^{\mathrm{C}}+\rho_{\mathrm{l}} F_{\mathrm{g}}^{\mathrm{C}}=\rho_{\mathrm{g}} \rho_{\mathrm{l}} v
$$

for the contact discontinuity (32).

Definition 1. A pair of numerical fluxes $F_{1}$ and $F_{\mathrm{g}}$ that satisfy (50) for the contact discontinuity (32) will in the following be termed "pressure preserving" fluxes.

In particular, we observe that the upwind fluxes (33) are pressure preserving. We now state the following lemma relevant for schemes obtained by using the hybrid mass fluxes (40) and (41).

Lemma 1. Let the hybrid fluxes (40) and (41) be constructed from pressure preserving fluxes $F_{k}^{C}$, and fluxes $F_{k}^{U}$ that reduce to the upwind fluxes (33) on a contact discontinuity of the form (32). Then the hybrid fluxes (40) and (41) also reduce to the upwind fluxes (33) on the contact discontinuity (32).

Proof. We consider the hybrid liquid mass flux (40) and assume that $v \geq 0$. Remembering that a subscript $j+1 / 2$ is assumed on the variables, we write the flux as

$$
F_{1}=\kappa\left(\alpha_{1} \frac{\partial \rho_{\mathrm{l}}}{\partial p}\left(\rho_{\mathrm{g}} F_{\mathrm{l}}^{\mathrm{C}}+\rho_{\mathrm{l}} F_{\mathrm{g}}^{\mathrm{C}}\right)+\rho_{\mathrm{l}} \alpha_{\mathrm{g}} \frac{\partial \rho_{\mathrm{g}}}{\partial p} F_{\mathrm{l}}^{\mathrm{U}}-\rho_{\mathrm{l}} \alpha_{\mathrm{l}} \frac{\partial \rho_{\mathrm{l}}}{\partial p} F_{\mathrm{g}}^{\mathrm{U}}\right)
$$

Using the required properties of $F_{k}^{\mathrm{C}}$ and $F_{k}^{\mathrm{U}}$ we obtain

$$
F_{\mathrm{l}}=\kappa\left(\alpha_{\mathrm{l}} \frac{\partial \rho_{\mathrm{l}}}{\partial p} \rho_{\mathrm{g}} \rho_{\mathrm{l}} v+\rho_{\mathrm{l}}^{2} \alpha_{\mathrm{g}} \frac{\partial \rho_{\mathrm{g}}}{\partial p}\left(\alpha_{\mathrm{l}}\right)_{\mathrm{L}} v-\rho_{\mathrm{g}} \rho_{\mathrm{l}} \alpha_{\mathrm{l}} \frac{\partial \rho_{\mathrm{l}}}{\partial p}\left(1-\left(\alpha_{\mathrm{l}}\right)_{\mathrm{L}}\right) v\right)=\rho_{\mathrm{l}}\left(\alpha_{\mathrm{l}}\right)_{\mathrm{L}} v
$$

where we have used that

$$
\rho_{j+1 / 2}=\rho_{j}=\rho_{j+1}
$$

which follows from the assumption of constant, uniform pressure. Spatial and phasic symmetry directly give the corresponding results for $F_{\mathrm{g}}$ and $v \leq 0$, completing the proof. 
In view of Lemma 1 we obtain the following result for the HCU scheme.

Proposition 1. The mass fluxes of the HCU scheme described in Section 5.4 reduce to the upwind fluxes (33) on a contact discontinuity of the form (32).

Proof. In view of Section 5.2 we know that the $F_{k}^{\mathrm{U}}$ components in the HCU scheme reduce to the upwind fluxes (33) on a contact discontinuity of the form (32). Thus, we only need to check that the $F_{k}^{\mathrm{C}}$ components given by (29) are pressure preserving in the sense of Definition 1 and then appeal to Lemma 1. Substituting constant pressure and velocities in (29) we get

$$
\begin{aligned}
\rho_{\mathrm{g}} F_{\mathrm{l}}^{\mathrm{C}}+\rho_{\mathrm{l}} F_{\mathrm{g}}^{\mathrm{C}}= & \rho_{\mathrm{g}} \rho_{\mathrm{l}}\left[\frac{v}{2}\left(\alpha_{\mathrm{l}, j}+\alpha_{\mathrm{l}, j+1}\right)+\frac{\Delta x}{4 \Delta t}\left(\alpha_{\mathrm{l}, j}-\alpha_{\mathrm{l}, j+1}\right)\right] \\
& +\rho_{\mathrm{g}} \rho_{\mathrm{l}}\left[\frac{v}{2}\left(\alpha_{\mathrm{g}, j}+\alpha_{\mathrm{g}, j+1}\right)+\frac{\Delta x}{4 \Delta t}\left(\alpha_{\mathrm{g}, j}-\alpha_{\mathrm{g}, j+1}\right)\right] \\
= & \rho_{\mathrm{g}} \rho_{\mathrm{l}}\left[\frac{v}{2}(1+1)+\frac{\Delta x}{4 \Delta t}(1-1)\right]=\rho_{\mathrm{g}} \rho_{\mathrm{l}} v .
\end{aligned}
$$

\subsection{Abgrall's principle}

According to the principle due to Abgrall $[1,18]$ it is desirable that the numerical scheme respects the following physical principle:

A flow, uniform in pressure and velocity must remain uniform in the same variables during its time evolution. In other words, if we had constant pressure and velocity everywhere in a flow at the time level $t^{n}$, then we will get the same pressure and velocity at the time $t^{n+1}$.

We now check if the HCU scheme obeys Abgrall's principle. Consequently, we assume that we have the contact discontinuity given by (32) and that it remains unchanged during the time interval $\left[t^{n}, t^{n+1}\right]$. In view of Proposition 1 and the fact that the convective fluxes of the momentum equations are based on an upwind scheme, we immediately conclude that the mass and momentum equations take the form

$$
\begin{aligned}
(\rho \alpha)_{j}^{n+1}= & (\rho \alpha)_{j}^{n}-\frac{\Delta t}{\Delta x}\left((\rho \alpha v)_{j+1 / 2}^{n}-(\rho \alpha v)_{j-1 / 2}^{n}\right) \\
v(\rho \alpha)_{j}^{n+1}= & v(\rho \alpha)_{j}^{n}-v \frac{\Delta t}{\Delta x}\left((\rho \alpha v)_{j+1 / 2}^{n}-(\rho \alpha v)_{j-1 / 2}^{n}\right) \\
& -(\Delta p)_{j}^{n} \frac{\Delta t}{2 \Delta x}\left(\alpha_{j+1}^{n}-\alpha_{j-1}^{n}\right)-\frac{\Delta t}{\Delta x}\left(p_{j+1 / 2}^{n+1}-p_{j-1 / 2}^{n+1}\right),
\end{aligned}
$$

where $(\rho \alpha v)_{j+1 / 2}^{n}$ is on the form (33). From (9) we conclude that $(\Delta p)_{j}^{n}=0$. Referring to (20) we also see that $p_{j+1 / 2}^{n+1}$ reduces to

$$
p_{j+1 / 2}^{n+1}=p-\kappa_{j+1 / 2}^{n} \frac{\Delta t}{\Delta x}\left[\rho_{\mathrm{l}} \rho_{\mathrm{g}} v\left(\alpha_{\mathrm{g}, j+1}-\alpha_{\mathrm{g}, j}\right)+\rho_{\mathrm{l}} \rho_{\mathrm{g}} v\left(\alpha_{\mathrm{l}, j+1}-\alpha_{\mathrm{l}, j}\right)\right]=p .
$$

Consequently, the pressure terms vanish and we can conclude that the HCU scheme satisfies Abgrall's principle.

\section{NumERICAL SIMULATIONS}

In the following, some selected numerical experiments will be presented. To highlight the accuracy, robustness and convergence properties of the HCU scheme, we will focus on some benchmark shock tube problems as well as a classical water faucet problem. Comparisons are made with a Roe scheme (see [8] for the implementation) and the AUSM type schemes considered in $[8,15]$. 


\subsection{Large relative velocity shock}

We consider an initial Riemann problem previously investigated in [5,8]. The initial states are given by

$$
\mathbf{W}_{\mathrm{L}}=\left[\begin{array}{c}
p \\
\alpha_{1} \\
v_{\mathrm{g}} \\
v_{\mathrm{l}}
\end{array}\right]=\left[\begin{array}{c}
265000 \\
0.71 \\
65 \\
1
\end{array}\right]
$$

and

$$
\mathbf{W}_{\mathrm{R}}=\left[\begin{array}{c}
p \\
\alpha_{\mathrm{l}} \\
v_{\mathrm{g}} \\
v_{\mathrm{l}}
\end{array}\right]=\left[\begin{array}{c}
265000 \\
0.7 \\
50 \\
1
\end{array}\right]
$$

No source terms are taken into account.

\subsubsection{Comparison between central and HCU scheme}

We first wish to illustrate the effect of introducing the hybrid mass flux obtained by combining the two different flux components $F_{k}^{\mathrm{C}}$ and $F_{k}^{\mathrm{U}}$ as described by (40) and (41). We assume a grid of 100 cells and a timestep of

$$
\frac{\Delta x}{\Delta t}=10^{3} \mathrm{~m} / \mathrm{s} .
$$

The reference solution was calculated by the Roe scheme on a grid of 10000 cells.

Results for the central and HCU scheme are given in Figure 1, at the time $T=0.1 \mathrm{~s}$. We note the following:

- The central scheme is robust but highly diffusive for the volume fraction wave.

- The HCU is significantly less dissipative than the central scheme, with no associated loss of stability.

\subsubsection{Comparison between HCU and Roe scheme}

The HCU scheme is compared to the Roe scheme in Figure 2. The HCU scheme is slightly more dissipative than the Roe scheme on the leftward (slowest) sonic wave, otherwise they are virtually identical. A comparison with the results presented in [8] demonstrates a significant advantage in robustness over the AUSMD/V approach for this problem.

\subsection{Modified large relative velocity shock}

We consider a modified version of the LRV shock, where we introduce a jump in the liquid velocity as well as a larger jump in volume fraction. This problem was studied as shock tube problem 2 in [8].

The initial states are given by

$$
\mathbf{W}_{\mathrm{L}}=\left[\begin{array}{c}
p \\
\alpha_{\mathrm{l}} \\
v_{\mathrm{g}} \\
v_{\mathrm{l}}
\end{array}\right]=\left[\begin{array}{c}
265000 \mathrm{~Pa} \\
0.7 \\
65 \mathrm{~m} / \mathrm{s} \\
10 \mathrm{~m} / \mathrm{s}
\end{array}\right]
$$

and

$$
\mathbf{W}_{\mathrm{R}}=\left[\begin{array}{c}
p \\
\alpha_{\mathrm{l}} \\
v_{\mathrm{g}} \\
v_{\mathrm{l}}
\end{array}\right]=\left[\begin{array}{c}
265000 \mathrm{~Pa} \\
0.1 \\
50 \mathrm{~m} / \mathrm{s} \\
15 \mathrm{~m} / \mathrm{s}
\end{array}\right]
$$



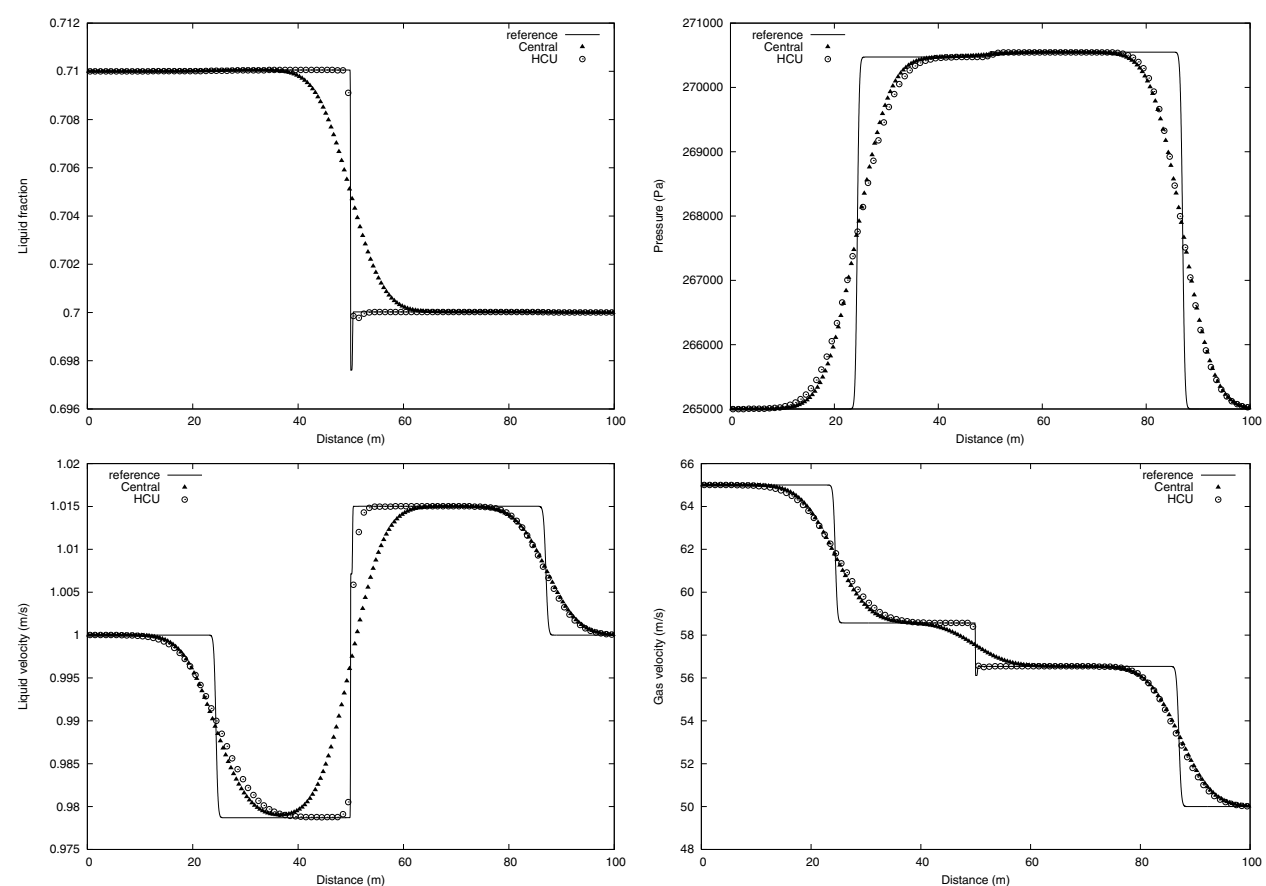

FiguRE 1. LRV shock tube problem, $T=0.1 \mathrm{~s}, 100$ grid cells. Central vs. HCU scheme. Top left: liquid fraction. Top right: pressure. Bottom left: liquid velocity. Bottom right: gas velocity.
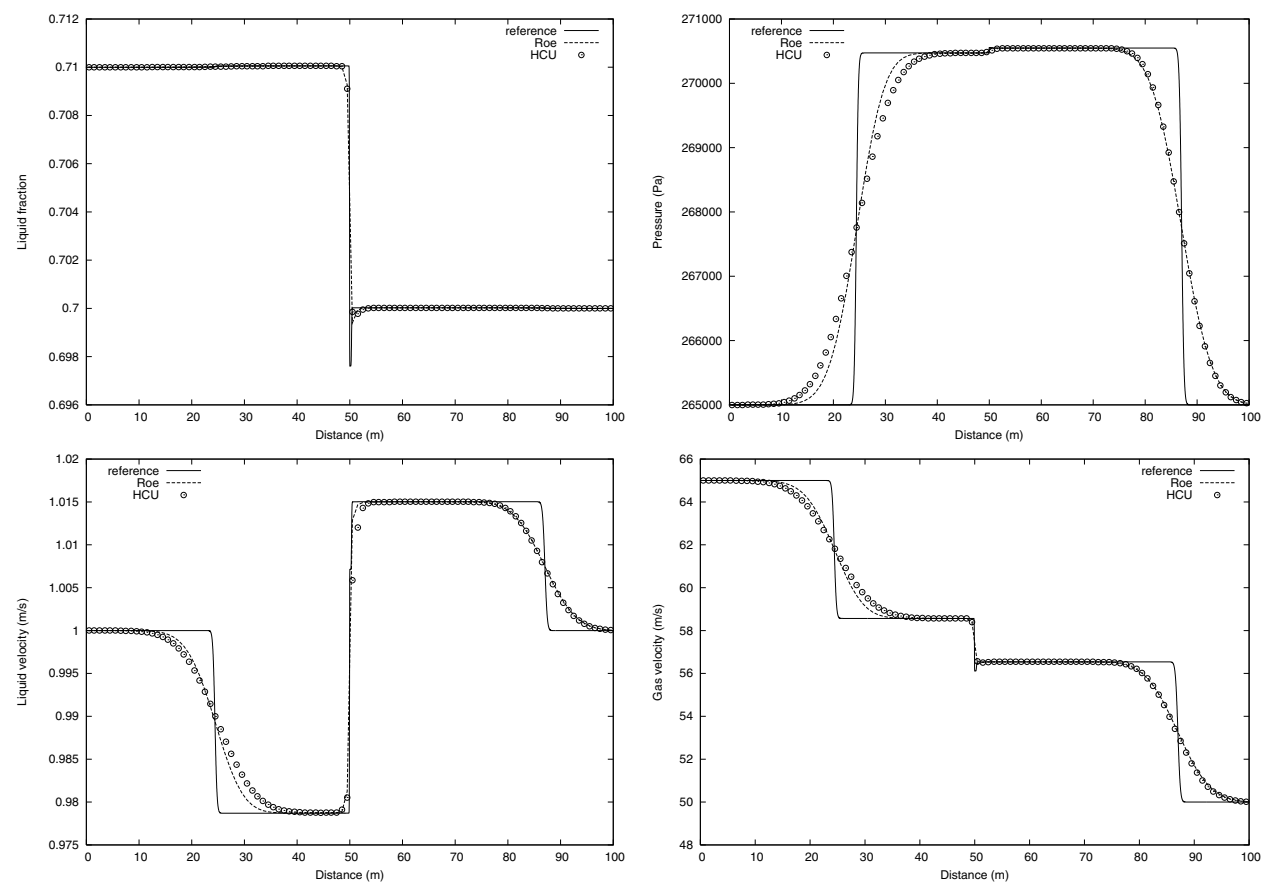

Figure 2. LRV shock tube problem, $T=0.1 \mathrm{~s}, 100$ grid cells. HCU vs. Roe scheme. Top left: liquid fraction. Top right: pressure. Bottom left: liquid velocity. Bottom right: gas velocity. 

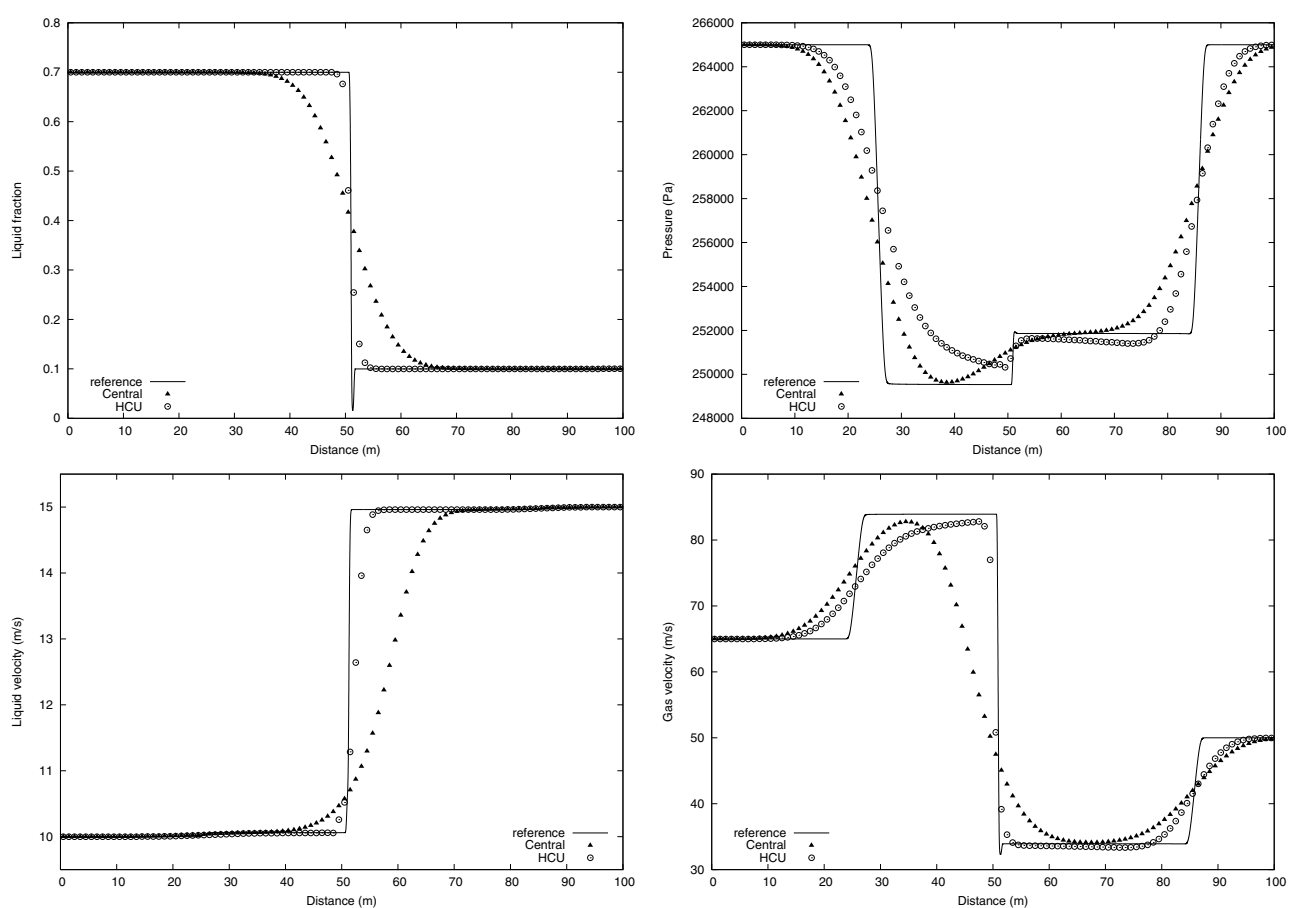

Figure 3. Modified LRV shock tube problem, $T=0.1 \mathrm{~s}, 100$ grid cells. Central vs. HCU scheme. Top left: liquid fraction. Top right: pressure. Bottom left: liquid velocity. Bottom right: gas velocity.

\subsubsection{Comparison between central and $H C U$ scheme}

Results after $T=0.1 \mathrm{~s}$ are given in Figure 3, using a grid of 100 cells and a timestep $\Delta x / \Delta t=750 \mathrm{~m} / \mathrm{s}$. The reference solution was calculated by the Roe scheme on a grid of 20000 cells.

Similar to what was observed in Section 7.1.1, both schemes yield a nonoscillatory approximation whereas the HCU scheme is more accurate.

\subsubsection{Comparison between $H C U$ and Roe scheme}

The HCU scheme is compared to the Roe scheme in Figure 4. As in Section 7.1.2, the only observable difference between the schemes is that HCU smears the leftward sonic wave slightly more than the Roe scheme.

\subsubsection{Convergence properties of the $H C U$ scheme}

In Figure 5 we investigate the effect of grid refinement for the HCU scheme. The plot of liquid fraction has been magnified, the legend refers to the number of visible grid cells.

The HCU scheme converges to the Roe reference solution is a monotone manner. A comparison with the results of [8] indicates that the HCU scheme is more accurate, and less oscillatory, than the AUSMD/V approach for this problem. 

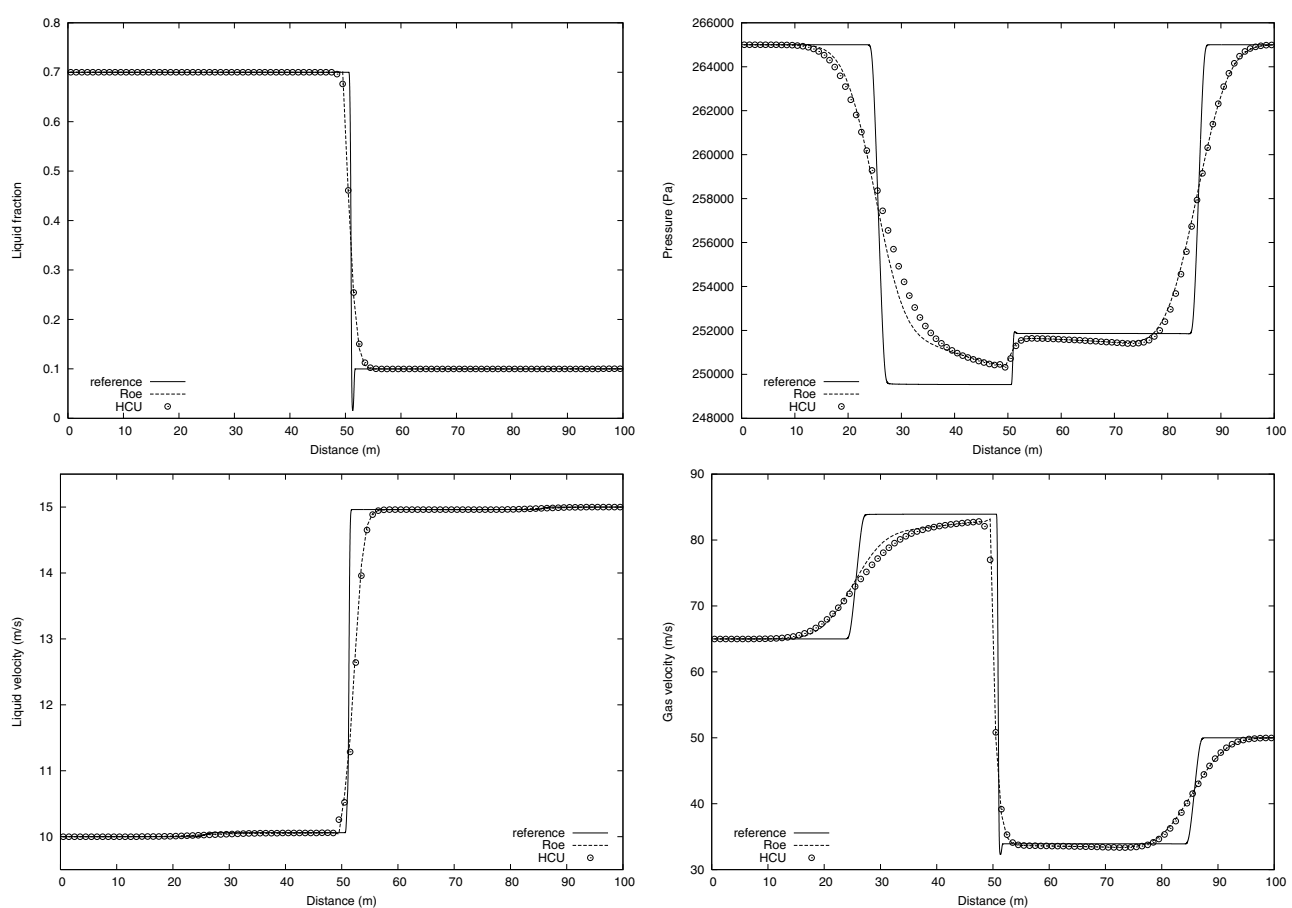

Figure 4. Modified LRV shock tube problem, $T=0.1 \mathrm{~s}, 100$ grid cells. HCU vs. Roe scheme. Top left: gas fraction. Top right: pressure. Bottom left: liquid velocity. Bottom right: gas velocity.

\subsection{Toumi's water-air shock}

We now consider an initial value problem of a kind introduced by Toumi [21] and investigated by Tiselj and Petelin [20] and Paillère et al. [15]. The initial states are given by

$$
\mathbf{W}_{\mathrm{L}}=\left[\begin{array}{c}
p \\
\alpha_{\mathrm{l}} \\
v_{\mathrm{g}} \\
v_{\mathrm{l}}
\end{array}\right]=\left[\begin{array}{c}
2 \times 10^{7} \mathrm{~Pa} \\
0.75 \\
0 \\
0
\end{array}\right]
$$

and

$$
\mathbf{W}_{\mathrm{R}}=\left[\begin{array}{c}
p \\
\alpha_{\mathrm{l}} \\
v_{\mathrm{g}} \\
v_{\mathrm{l}}
\end{array}\right]=\left[\begin{array}{c}
1 \times 10^{7} \mathrm{~Pa} \\
0.9 \\
0 \\
0
\end{array}\right]
$$

Again no source terms are taken into account. Following Paillère et al. [15], we modify the interfacial pressure correction (9) for this problem, setting $\sigma=2$. By this modification, the velocity difference between the volume fraction waves is magnified and the full wave structure of the model becomes more pronounced.

\subsubsection{Comparison between central and $\mathrm{HCU}$ scheme}

Results after $T=0.08 \mathrm{~s}$ are given in Figure 6, using a grid of 200 cells and a timestep $\Delta x / \Delta t=1000 \mathrm{~m} / \mathrm{s}$. The reference solution was calculated by the HCU scheme using a grid of 10000 cells.

The central scheme is fully nonoscillatory. While being significantly more accurate, the HCU scheme here introduces a slight overshoot in volume fraction. 

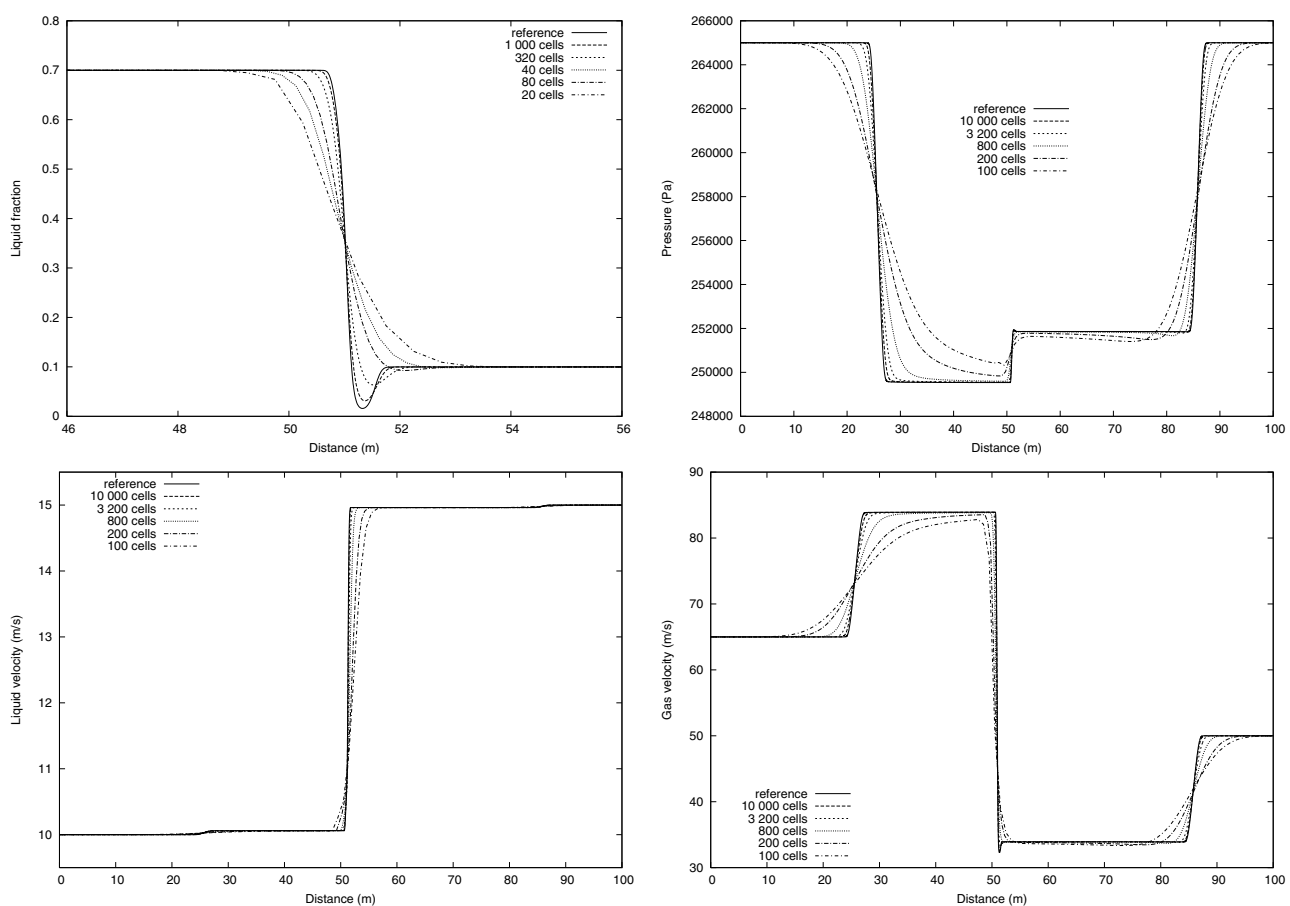

FIGURE 5. Modified LRV shock tube problem, $T=0.1 \mathrm{~s}$. Grid refinement for the HCU scheme. Top left: liquid fraction. Top right: pressure. Bottom left: liquid velocity. Bottom right: gas velocity.

\subsubsection{Convergence properties of the $\mathrm{HCU}$ scheme}

In Figure 7 we study the convergence of the HCU scheme as the grid is refined. A small overshoot is observed in volume fraction and liquid velocity for coarse grids, these overshoots decay rapidly with grid refinement.

For the initial conditions $v_{\mathrm{g}}=v_{\mathrm{l}}$, the model becomes non-diagonalizable (see for instance [8]). Hence the Roe scheme is not applied for this test case. However, our numerical results are comparable with the results reported by Paillère et al. [15], obtained by an $\mathrm{AUSM}^{+}$scheme with a pressure diffusion term.

\subsection{Water faucet problem}

We finally consider a benchmark faucet flow problem proposed by Ransom [16], which has been extensively studied $[4,8,14,15,22,23]$.

We consider a vertical pipe of length $12 \mathrm{~m}$ with the initial uniform state

$$
\mathbf{W}=\left[\begin{array}{c}
p \\
\alpha_{1} \\
v_{\mathrm{g}} \\
v_{\mathrm{l}}
\end{array}\right]=\left[\begin{array}{c}
10^{5} \mathrm{~Pa} \\
0.8 \\
0 \\
10 \mathrm{~m} / \mathrm{s}
\end{array}\right]
$$

Gravity is the only source term taken into account, i.e. in the framework of (4) and (5) we have

$$
Q_{k}=g \rho_{k} \alpha_{k},
$$



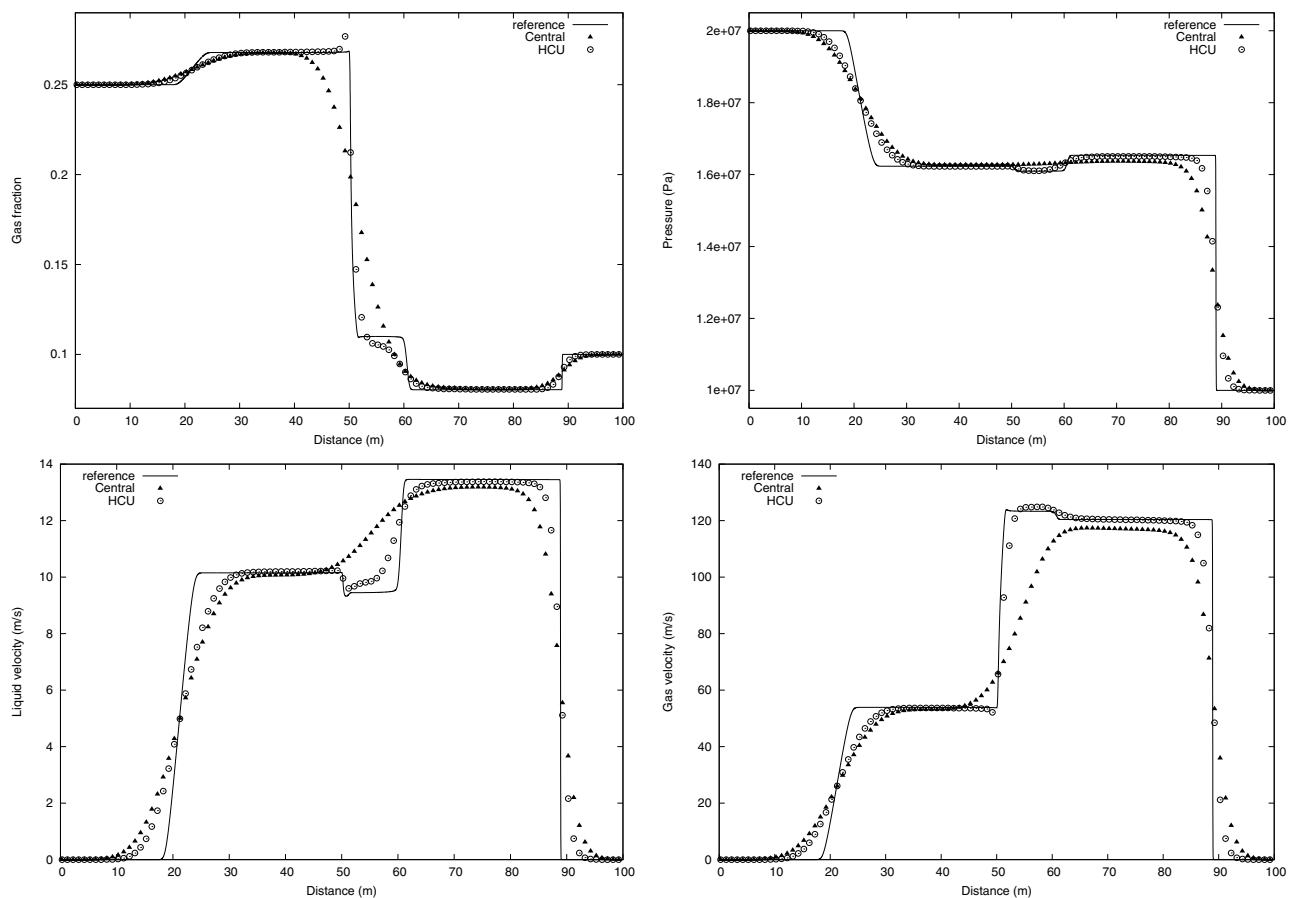

Figure 6. Toumi's shock tube problem, $T=0.08,200$ grid cells. Central vs. HCU scheme. Top left: gas fraction. Top right: pressure. Bottom left: liquid velocity. Bottom right: gas velocity.

with $g$ being the acceleration of gravity. At the inlet we have the constant conditions $\alpha_{1}=0.8, v_{1}=10 \mathrm{~m} / \mathrm{s}$ and $v_{\mathrm{g}}=0$. At the outlet the pipe is open to the ambient pressure $p=10^{5} \mathrm{~Pa}$. The remaining variables at the boundaries are determined by simple extrapolation.

A "contact" discontinuity in the volume fraction will arise as the liquid falls under the acceleration of gravity. It is possible to express an approximate solution in analytical form: $[8,15,23]$

$$
\begin{gathered}
v_{\mathrm{l}}(x, t)= \begin{cases}\sqrt{v_{0}^{2}+2 g x} & \text { for } x<v_{0} t+\frac{1}{2} g t^{2} \\
v_{0}+g t & \text { otherwise. }\end{cases} \\
\alpha_{1}(x, t)= \begin{cases}\alpha_{0}\left(1+2 g x v_{0}^{-2}\right)^{-1 / 2} & \text { for } x<v_{0} t+\frac{1}{2} g t^{2} \\
\alpha_{0} & \text { otherwise. }\end{cases}
\end{gathered}
$$

The parameters $\alpha_{0}=0.8$ and $v_{0}=10 \mathrm{~m} / \mathrm{s}$ are the initial states.

\subsubsection{Comparison between $\mathrm{HCU}$ and Roe scheme}

In Figure 8 the HCU scheme is compared to the Roe scheme for $T=0.6 \mathrm{~s}$ on a grid of 120 computational cells. The timestep $\Delta x / \Delta t=10^{3} \mathrm{~m} / \mathrm{s}$ is used. The pressure and gas velocity references were calculated using the Roe scheme on a grid of 12000 cells, for the volume fraction and liquid velocity the approximate analytical expressions were used. The results of the HCU and Roe scheme are virtually identical. 

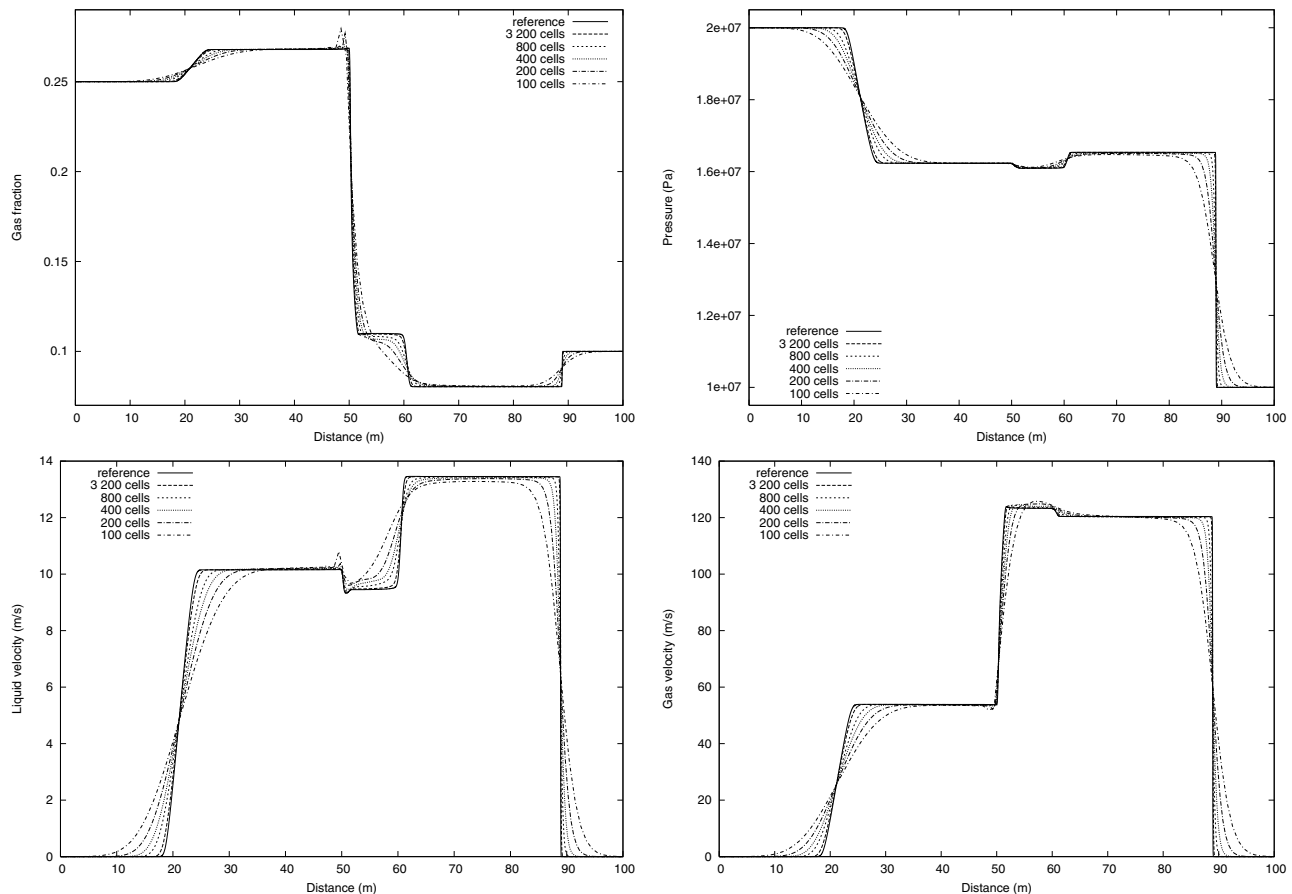

Figure 7. Toumi's shock tube problem. Grid refinement for the HCU scheme. Top left: gas fraction. Top right: pressure. Bottom left: liquid velocity. Bottom right: gas velocity.
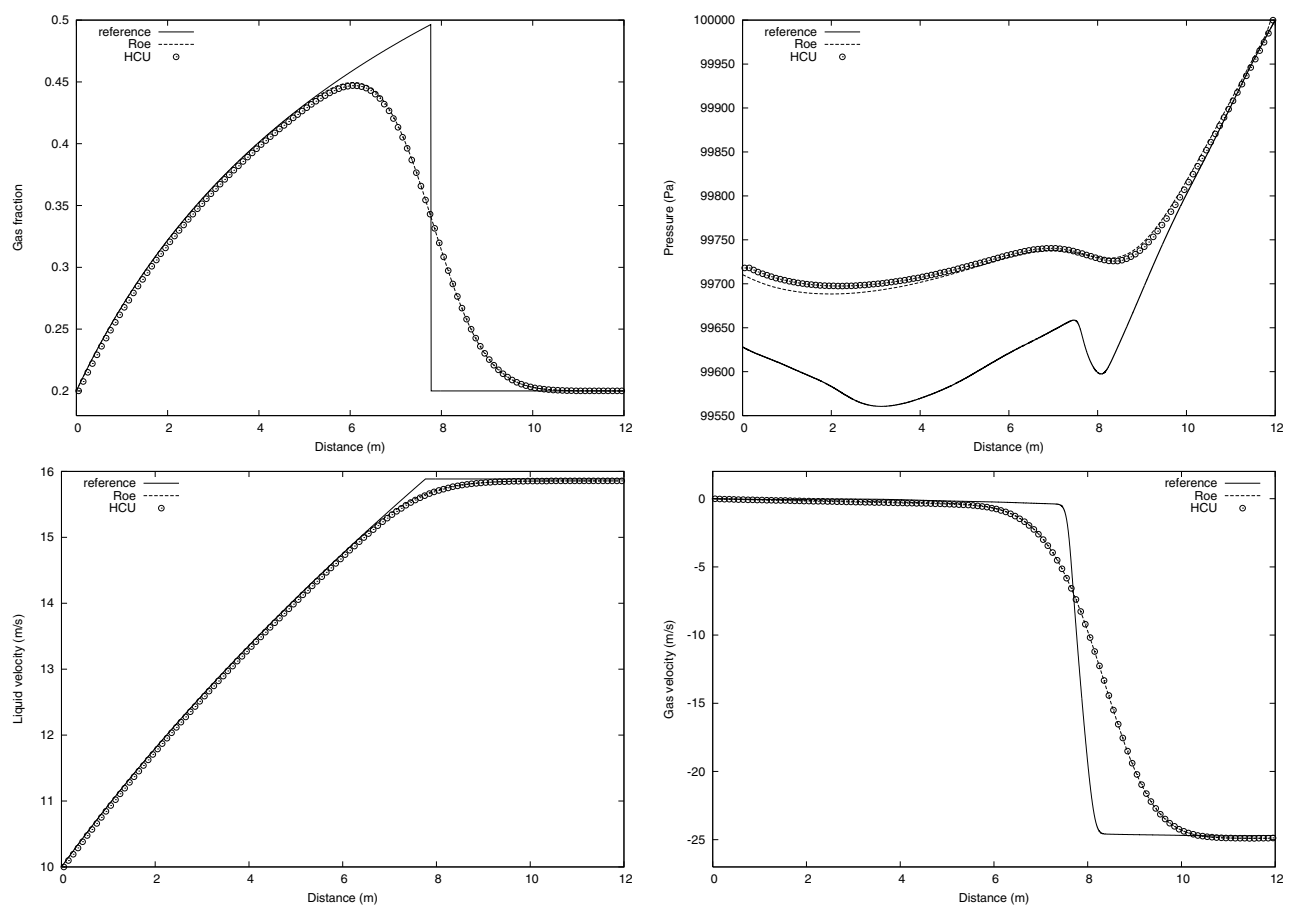

Figure 8. Water faucet problem, $T=0.6 \mathrm{~s}, 120$ grid cells. HCU vs. Roe scheme. Top left: gas fraction. Top right: pressure. Bottom left: liquid velocity. Bottom right: gas velocity. 

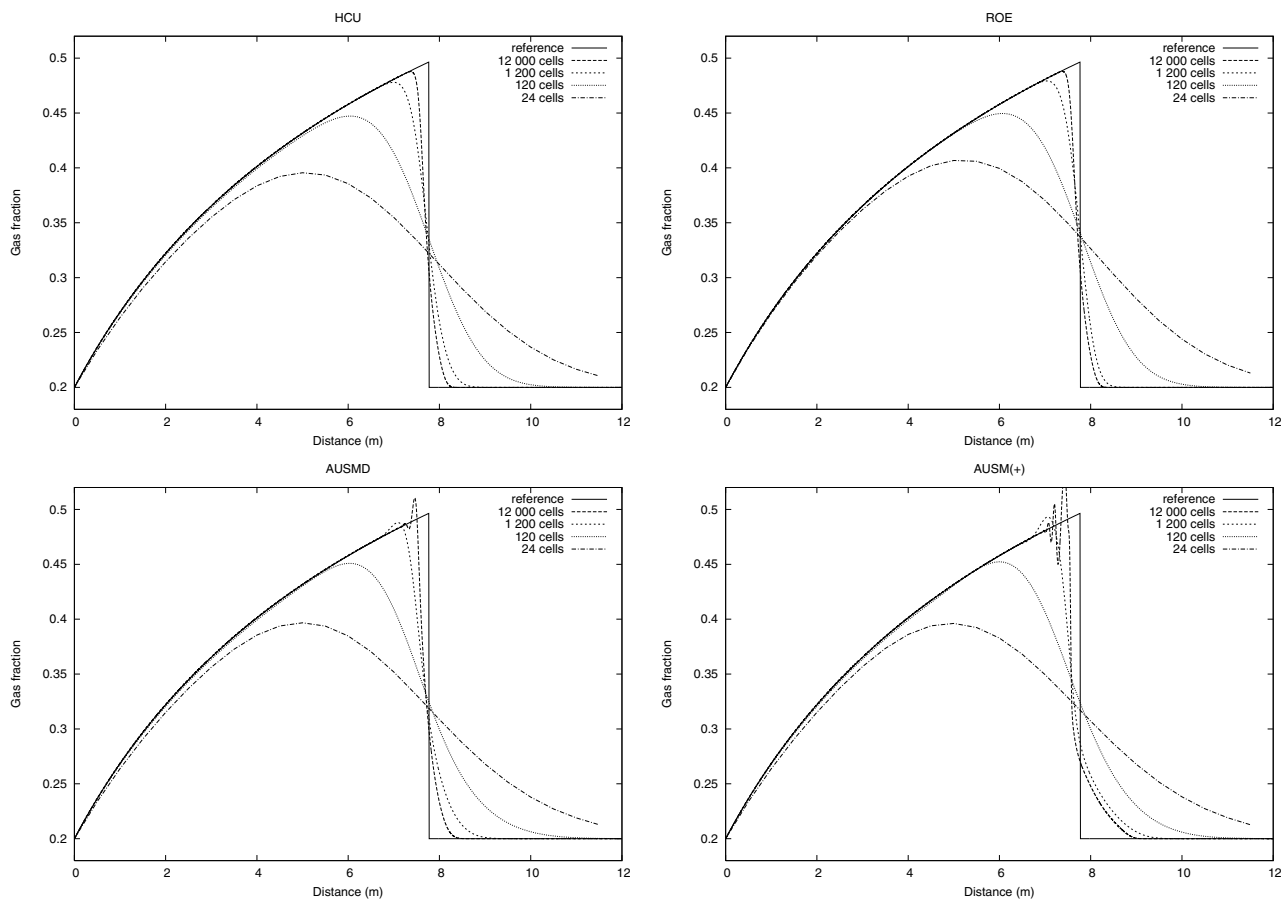

FiguRE 9. Water faucet problem, $T=0.6 \mathrm{~s}$. Resolution of volume fraction. Top left: grid refinement for the HCU scheme. Top right: grid refinement for the Roe scheme. Bottom left: grid refinement for the AUSMD scheme. Bottom right: grid refinement for the AUSM+ ${ }^{+}$scheme.

\subsubsection{Convergence properties of different schemes}

In Figure 9 we investigate how different schemes converge to the expected analytical solution as the grid is refined. The HCU, Roe and AUSM type schemes of $[8,15]$ are considered.

Both the Roe and HCU schemes seem to monotonely approach the expected analytical solution. However, oscillations and overshoots are observed for both AUSM type schemes - supporting the belief that the more sophisticated dissipation mechanism of HCU, expressed through the flux mixing (40) and (41) together with the use of the pressure evolution equation, is inherently more robust than the AUSM strategy.

\section{Summary}

We have presented a framework for constructing accurate and robust hybrid central-upwind schemes for an isothermal two-phase flow model. A main idea has been to develop a pressure evolution equation which provides us with a numerical pressure flux which yields a highly robust treatment of the nonlinearity associated with the pressure law $p\left(m_{\mathrm{g}}, m_{\mathrm{l}}\right)$. Then, in order to improve the accuracy of mass fronts we modify the convective fluxes. More precisely, we have derived the expressions (40) and (41) for hybridizing a central and upwind mass flux, by enforcing robustness (central scheme) on the pressure variable and accuracy (upwind scheme) on the volume fraction variable.

The HCU scheme presented in this paper possesses an accuracy and robustness comparable to a Roe scheme. In particular, the HCU does not involve solving a local Riemann problem by eigenstructure decomposition and is therefore superior to the Roe scheme when it comes to computational efficiency.

Further extensions that naturally can be explored within the framework developed in this paper are incorporation of source terms, variable sections, extension to several components and several phases. An important step that we should consider in the future is inclusion of full EOS where $\rho=\rho(p, e)(e$ is the energy), and 
corresponding energy equations. This will require a suitable modification of the pressure evolution equation as well as the differential relations for the construction of hybrid central-upwind mass fluxes.

Acknowledgements. The authors thank the Research Council of Norway for financial support. We also thank the reviewers for clarifying some issues through their insightful comments.

\section{REFERENCES}

[1] R. Abgrall, How to prevent pressure oscillations in multicomponent flow calculations. J. Comput. Phys. 125 (1996) 150-160.

[2] F. Barre et al., The CATHARE code strategy and assessment. Nucl. Eng. Des. 124 (1990) 257-284.

[3] K.H. Bendiksen, D. Malnes, R. Moe and S. Nuland, The dynamic two-fluid model OLGA: Theory and application, in SPE Prod. Eng. 6 (1991) 171-180.

[4] F. Coquel, K. El Amine, E. Godlewski, B. Perthame and P. Rascle, A numerical method using upwind schemes for the resolution of two-phase flows. J. Comput. Phys. 136 (1997) 272-288.

[5] J. Cortes, A. Debussche and I. Toumi, A density perturbation method to study the eigenstructure of two-phase flow equation systems. J. Comput. Phys. 147 (1998) 463-484.

[6] S. Evje and K.K. Fjelde, Hybrid flux-splitting schemes for a two-phase flow model. J. Comput. Phys. 175 (2002) 674-701.

[7] S. Evje and K.K. Fjelde, On a rough ausm scheme for a one-dimensional two-phase flow model. Comput. Fluids 32 (2003) $1497-1530$.

[8] S. Evje and T. Flåtten, Hybrid flux-splitting schemes for a common two-fluid model. J. Comput. Phys. 192 (2003) $175-210$.

[9] S. Evje and T. Flåtten, Weakly implicit numerical schemes for a two-fluid model. SIAM J. Sci. Comput., accepted.

[10] T. Flåtten, Hybrid flux-splitting schemes for numerical resolution of two-phase flows. Dr.ing.-thesis, Norwegian University of Science and Technology (2003) 114.

[11] M. Larsen, E. Hustvedt, P. Hedne and T. Straume, PeTra: A novel computer code for simulation of slug flow, in SPE Annual Technical Conference and Exhibition, SPE 38841 (October 1997) 1-12.

[12] M.-S. Liou, A sequel to AUSM: AUSM(+). J. Comput. Phys. 129 (1996) 364-382.

[13] Y.Y. Niu, Simple conservative flux splitting for multi-component flow calculations. Num. Heat Trans. 38 (2000) $203-222$.

[14] Y.Y. Niu, Advection upwinding splitting method to solve a compressible two-fluid model. Internat. J. Numer. Methods Fluids 36 (2001) 351-371.

[15] H. Paillère, C. Corre and J.R.G. Cascales, On the extension of the AUSM+ scheme to compressible two-fluid models. Comput. Fluids 32 (2003) 891-916.

[16] V.H. Ransom, Numerical bencmark tests. Multiphase Sci. Tech. 3 (1987) 465-473.

[17] V.H. Ransom et al., RELAP5/MOD3 Code Manual, NUREG/CR-5535, Idaho National Engineering Laboratory (1995).

[18] R. Saurel and R. Abgrall, A multiphase Godunov method for compressible multifluid and multiphase flows. J. Comput. Phys. 150 (1999) 425-467.

[19] E. Tadmor, Numerical viscosity and the entropy condition for conservative difference schemes. Math. Comp. 168 (1984) 369-381.

[20] I. Tiselj and S. Petelin, Modelling of two-phase flow with second-order accurate scheme. J. Comput. Phys. 136 (1997) 503-521.

[21] I. Toumi, An upwind numerical method for two-fluid two-phase flow models. Nuc. Sci. Eng. 123 (1996) 147-168.

[22] I. Toumi and A. Kumbaro, An approximate linearized riemann solver for a two-fluid model. J. Comput. Phys. 124 (1996) $286-300$.

[23] J.A. Trapp and R.A. Riemke, A nearly-implicit hydrodynamic numerical scheme for two-phase flows. J. Comput. Phys. 66 (1986) 62-82.

[24] Y. Wada and M.-S. Liou, An accurate and robust flux splitting scheme for shock and contact discontinuities. SIAM J. Sci. Comput. 18 (1997) 633-657. 